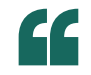

The short fermentation time ... and solvent-free lipid recovery improve economic and ecological process parameters

\title{
Palms make way for oleaginous yeast
}

Palm oil is useful because its low unsaturation confers oxidative stability desirable in kitchens and energy density desirable in fuels. The economic cost of palm oil is low, but the same cannot be said for its environmental cost, which includes habitat destruction and a net release of greenhouse gases. Triglycerides comparable to palm oil can be found in yeast grown on sugars produced from waste biomass and acetic acid from bacterial fermentation of $\mathrm{CO} / \mathrm{H}_{2}$ or $\mathrm{CO}_{2} / \mathrm{H}_{2}$ mixtures. Although sustainable, these feedstocks can be expensive, and yeast oils form in low abundance and must be extracted with an organic solvent. In view of this, Mahmoud Masri, Daniel Garbe, Norbert Mehlmer and Thomas Brück describe in Energy \& Environmental Science a sustainable and economical process in which yeast cells, cultured in a nitrogen-rich medium, are enzymatically digested to liberate high yields of biodiesel-ready oils.

When yeast has plenty of C, N, P and $\mathrm{S}$, it readily produces nucleic acids, proteins and polysaccharides, the latter two constituting a large fraction of the biomass of what is a rapidly growing culture. When the heteroatoms are in short supply, yeast instead expresses fatty acid synthases to store $\mathrm{C}$, via acetyl coenzyme A, as triglycerides that can be converted into other biomass when heteroatoms are available. To produce biomass quickly but not at the expense of triglycerides, Brück's team studied Cutaneotrichosporon oleaginosus, an oleaginous yeast that can comprise 80-90\% triglyceride by dry weight (for reference, baker's yeast is $<10 \%$ triglyceride). Culturing C. oleaginosus with different concentrations of sugars, acetic acid and yeast extract (a source of nitrogen) gave different profiles of total biomass and lipid mass fraction over time. Although some conditions result in diauxic yeast growth (phases of low and high triglyceride production), using glucose and acetic acid with nitrogen in abundance results in high monoauxic growth, a desirable regime in which lipid and biomass production are decoupled. This high-yielding fermentation in aerated, stirred tanks has a smaller spatial footprint than a palm plantation and is not subject to seasonal variability.

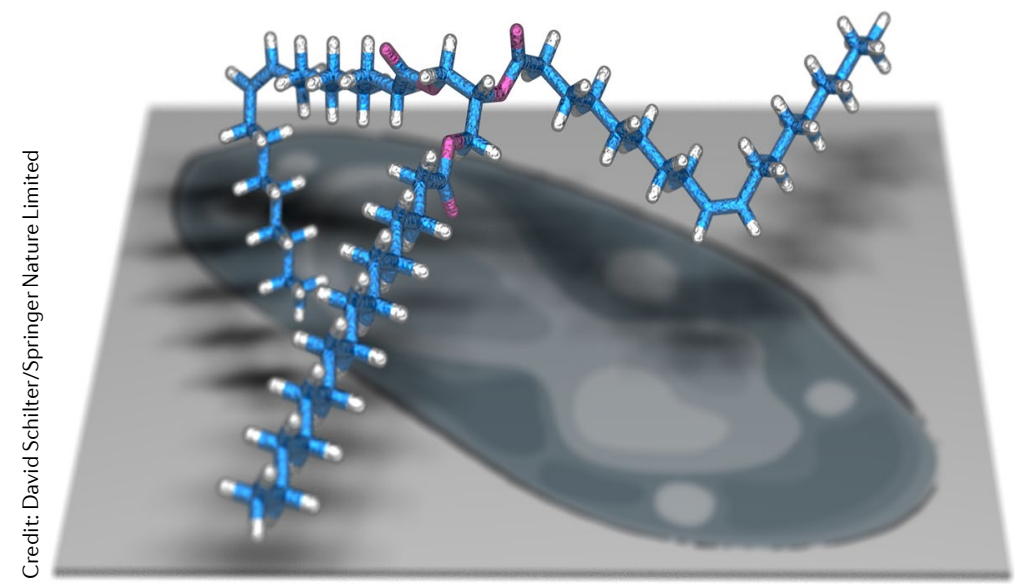

A fraction of the C. oleaginosus culture was periodically harvested and the cell walls (comprising chitin, glucans and proteins) digested using extracellular hydrolases expressed in Trichoderma reesei, grown in a separate vessel. The C. oleaginosus lysate comprises a biomass phase used to feed $T$. reese $i$ and an aqueous phase that is recycled to sustain the remaining C. oleaginosus, which can be further supported with a brown-algae-derived feed instead of glucose. A third gravimetrically separable oil phase (a mixture similar to palm oil), rich in triglycerides of oleic $\left(\mathrm{C}_{18}\right.$, monounsaturated $)$ and palmitic acid ( $\mathrm{C}_{16}$, saturated), is produced that can be hydrolysed to produce fatty acids for biodiesel use. "The short fermentation time (enabled by simultaneous lipid and biomass production) and solvent-free lipid recovery improve economic and ecological process parameters," notes Masri. Techno-economic analyses predict that the yeast oil (US\$1.6 $\mathrm{kg}^{-1}$ ) would be cheaper than eco-certified palm oil (US\$2.1 $\mathrm{kg}^{-1}$ ) and result in lower $\mathrm{CO}_{2}$ emissions.

It is remarkable how monoauxic growth with continually high triglyceride production can occur even in media with high $\mathrm{N}: \mathrm{C}$ ratios. Brück and colleagues note that, regardless of the $\mathrm{N}: \mathrm{C}$ ratio, C. oleaginosus expresses acetate coenzyme A ligase to directly convert acetic acid into acetyl coenzyme A. This enables fast and selective triglyceride production because fatty acid synthases from $C$. oleaginosus are particularly active. "We are working on a full proteomic and genomic analysis to identify these enzymes," concludes Masri. A deeper understanding of the cellular systems biology would help improve an already promising system.

David Schilter

ORIGINAL ARTICLE Masri, M. A. et al. A sustainable, high-performance process for the economic production of waste-free microbial oils that can replace plant-based equivalents. Energy Environ. Sci. https://doi.org/10.1039/C9EE00210C (2019) 